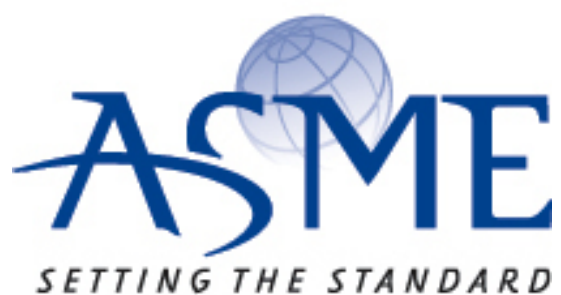

SETTING THE STANDARD

\section{American Society of Mechanical Engineers}

ASME Accepted Manuscript Repository

Institutional Repository Cover Sheet

ASME Paper Title: A machine learning approach to predicting the heat convection and thermodynamics of an

external flow of hybrid nanofluid

Alizadeh, R., Abad, J. M. N., Fattahi, A., Mohebbi, M. R., Doranehgard, M. H., Li, L. K.B., Alhajri,

Authors:

E. and Karimi, $\mathrm{N}$.

ASME Journal Title: Journal of Energy Resources Technology

Date of Publication (VOR* Online)

Volume/Issue _143(7) 23.12.2020

ASME Digital Collection URL: https://asmedigitalcollection.asme.org/

DOI:

*VOR (version of record) 


\title{
A machine learning approach to predicting the heat convection and thermodynamics of an external flow of hybrid nanofluid
}

\author{
Rasool Alizadeh ${ }^{1}$, Javad Mohebbi Najm Abad ${ }^{2}$, Abolfazl Fattahi ${ }^{3}$, Mohamad Reza Mohebbi ${ }^{2}$, \\ Mohammad Hossein Doranehgard ${ }^{4 *}$, Larry K.B. Li $^{5}$, Ebrahim Alhajri ${ }^{6}$, Nader Karimi ${ }^{7,8}$ \\ ${ }^{1}$ Department of Mechanical Engineering, Quchan Branch, Islamic Azad University, Quchan, Iran \\ ${ }^{2}$ Department of Computer Engineering, Quchan Branch, Islamic Azad University, Quchan, Iran \\ ${ }^{3}$ Department of Mechanical Engineering, University of Kashan, Kashan, Iran \\ ${ }^{4}$ Department of Civil and Environmental Engineering, School of Mining and Petroleum Engineering, University \\ of Alberta, Edmonton, Alberta, T6G 1H9, Canada \\ ${ }^{5}$ Department of Mechanical and Aerospace Engineering, Hong Kong University of Science and Technology, \\ Clear Water Bay, Hong Kong \\ ${ }^{6}$ Department of Mechanical Engineering, Khalifah University, Abu Dahabi, UAE \\ ${ }^{7}$ School of Engineering and Materials Science, Queen Mary University of London, London E1 4NS, United \\ Kingdom \\ ${ }^{8}$ James Watt School of Engineering, University of Glasgow, Glasgow G12 8QQ, United Kingdom \\ *Corresponding author: doranehg@ualberta.ca
}

\begin{abstract}
This study numerically investigates heat convection and entropy generation in a hybrid nanofluid $\left(\mathrm{Al}_{2} \mathrm{O}_{3}-\mathrm{Cu}\right.$-water) flowing around a cylinder embedded in porous media. An artificial-neural-network is used for predictive analysis, in which numerical data are generated to train an intelligence algorithm and to optimize the prediction errors. Results show that the heat transfer of the system increases when the Reynolds number, permeability parameter, or volume fraction of nanoparticles increases. However, the functional forms of these dependencies are complex. In particular, increasing the nanoparticle concentration is found to have a non-monotonic effect on entropy generation. The simulated and predicted data are subjected to particle swarm optimization to produce correlations for the shear stress and Nusselt number. This work demonstrates the capability of artificial intelligence algorithms in predicting the thermohydraulics and thermodynamics of thermal and solutal systems.
\end{abstract}

\section{Nomenclature}

\begin{tabular}{|l|l|l|l|}
\hline $\begin{array}{l}A_{1}, A_{2}, A_{3}, A_{4} \\
A_{5}\end{array}$ & Constants & $R e$ & $\begin{array}{l}\text { Freestream Reynolds number } R e= \\
\frac{\bar{k} \cdot a^{2}}{2 v}\end{array}$ \\
\hline$a$ & Cylinder radius & $\dot{S}_{g e n}^{\prime \prime \prime}$ & Rate of entropy generation \\
\hline ANN & Artificial neural network & $\dot{S}_{0}^{\prime \prime \prime}$ & $\begin{array}{l}\text { Characteristic entropy generation } \\
\text { rate }\end{array}$ \\
\hline$B e$ & Bejan number & $T$ & Temperature \\
\hline$B r$ & Brinkman number $B r=\frac{\mu_{f}(\bar{k} \cdot a)^{2}}{k_{f}\left(T_{w}-T_{\infty}\right)}$ & $u, w$ & $\begin{array}{l}\text { Velocity components along }(r-z)- \\
\text { axis }\end{array}$ \\
\hline$C_{p}$ & Specific heat at constant pressure & $z$ & Axial coordinate \\
\hline$f(\eta)$ & Radial velocity function & Greek symbols \\
\hline$f^{\prime}(\eta)$ & Axial velocity function & $\alpha$ & Thermal diffusivity \\
\hline GA & Genetic algorithm & $\eta$ & Similarity variable, $\eta=\left(\frac{r}{a}\right)^{2}$ \\
\hline
\end{tabular}




\begin{tabular}{|c|c|c|c|}
\hline$h$ & Heat transfer coefficient & $\theta(\eta)$ & Non-dimensional temperature \\
\hline ICA & Imperialist competition algorithm & $\lambda$ & Permeability parameter, $\lambda=\frac{a^{2}}{4 k_{1}}$ \\
\hline$k$ & Thermal conductivity & $\sigma$ & Shear stress \\
\hline $\bar{k}$ & Freestream strain rate & $\varepsilon$ & Porosity \\
\hline$k_{1}$ & Permeability of the porous medium & $\Lambda$ & $\begin{array}{l}\text { Non-dimensional temperature } \\
\text { difference } \Lambda=\frac{\left(T_{w}-T_{\infty}\right)}{T_{\infty}}\end{array}$ \\
\hline$m$ & Shape factor & $\mu$ & Dynamic viscosity \\
\hline MLP & Multi-layer perception & $v$ & Kinematic viscosity \\
\hline$N_{G}$ & Entropy generation number $N_{G}=\frac{\dot{S}_{g e n}{ }^{\prime \prime \prime}}{\dot{S}_{0}{ }^{\prime \prime \prime}}$ & $\rho$ & Fluid density \\
\hline $\mathrm{Nu}$ & Nusselt number & $\phi_{1}, \phi_{2}$ & Volume fraction of nanoparticles \\
\hline$p$ & Pressure of fluid & \multicolumn{2}{|c|}{ Subscripts } \\
\hline$P$ & Pressure of fluid (non-dimensional) & $w$ & External wall of the cylinder \\
\hline$P_{0}$ & Initial fluid pressure & $\infty$ & Far field \\
\hline $\operatorname{Pr}$ & Prandtl number & $h n f$ & Hybrid nanofluid \\
\hline PSO & Particle swarm optimization & $n f$ & Nanofluid \\
\hline$q_{w}$ & Heat flux on the wall & $f$ & Fluid \\
\hline$r$ & Radial coordinate & & \\
\hline
\end{tabular}

\section{Introduction}

The wide applicability and potential of hybrid nanofluids and porous media have led to a growing interest in combining them to achieve higher rates of heat transfer [1,2]. This has intensified the need to better understand and predict the characteristics of such combined systems, which in turn calls for parametric studies. However, heat transfer problems involving nanofluids and porous media often involve a large number of governing parameters [3-5]. This complicates the analysis, requiring a very large number of computationally costly simulations. The present study aims to address this issue by leveraging techniques from machine learning and combining them with computational fluid dynamics.

The general problem of heat transfer and entropy generation in nanofluid-porous-media systems has received considerable attention in the literature. For example, Ting et al. [6] investigated the entropy generation and heat transfer of a nanofluid $\left(\mathrm{Al}_{2} \mathrm{O}_{3}\right.$-water) moving through a porous microchannel. They found that entropy generation is mostly attributed to wall-heat generation, and that irreversibilities are dependent on the degree of symmetric heating, the Reynolds number, and the size and concentration of nanoparticles. Those researchers [6] also found that differences in the predictions between non-equilibrium and local-thermal-equilibrium models can be reduced by adding heat to the walls or by introducing nanoparticles to the base fluid. The work of Ting et al. [6] was later extended to microchannels with thick walls [7-9], demonstrating that such a geometry can contribute significantly to entropy generation.

Using numerical simulations, Kefayati [10] analyzed the heat transfer and thermodynamics of a nonNewtonian nanofluid flowing in a porous cavity. They found that increasing the volume fraction of 
nanoparticles, the Rayleigh number or the Darcy number can increase the Nusselt number and enhance entropy generation. In a similar study involving a Newtonian nanofluid, Hoseinpour et al. [11] found that larger Darcy and Rayleigh numbers can cause a flow to penetrate more readily into porous media, thus enhancing heat transfer. The total entropy generated was found to increase with porosity, as the fluid friction rose.

Siavashi et al. [12] numerically simulated a cavity of $\mathrm{Cu}$-water nanofluid equipped with porous fins. They found that the deployment of fins at large Darcy numbers can improve heat transfer by convection but impaired entropy generation, while a lower concentration of nanoparticles was found to lead to enhanced heat transfer. Flow friction was shown to be the main source of energy loss. To explore the sedimentation of $\mathrm{Al}_{2} \mathrm{O}_{3}$ nanoparticles in water, Baghsaz et al. [13] performed transient numerical simulations of a porous cavity. During the sedimentation stage, the Nusselt number was found to fluctuate as a result of deformations in the flow streamlines. Both the heat transfer and irreversibility were reduced in low-permeability enclosures.

Hussain et al. [14] examined the mixed convection of nanofluids in a chemically-reactive porous cavity. They showed that heat transfer increased with increasing volume fraction of nanoparticles, Darcy number and Richardson number, but that it decreased with increasing Lewis number and chemical reaction rate. The heat transfer mechanism responded to higher values of entropy generation, which increased with Richardson number.

Various studies have been conducted to investigate entropy generation in microchannel heat sinks filled with nanofluids, including those subjected to a magnetic field [15-18]. It was demonstrated that irreversibilities weakened with the introduction of a magnetic field [8]. Entropy generation near the walls was found to be the most important source of irreversibility in microchannels $[15,16]$. In some cases, Brownian motion was identified as the dominant process in the energy loss of closed tanks [18]. In those cases, the trends in heat transfer and entropy generation were in the same direction [16]. In such problems, there are many governing parameters, including the Reynolds number, Hartmann number, porosity, nanoparticle diameter, radiation number and volume fraction. Therefore, optimization methods should be used to identify a balanced trade-off between heat transfer and irreversibility [16,17]. Mansour et al. [19] examined the magnetohydrodynamic free-convection of a nanofluid $\left(\mathrm{Al}_{2} \mathrm{O}_{3}-\mathrm{Cu}\right.$-water) in a porous enclosure. The Nusselt number and entropy generated were found to decrease in response to a decreasing volume fraction of nanoparticles and a strengthening magnetic field.

As the number of governing parameters rises, so does the dimensionality of the system, making it more difficult to gain a complete understanding of the problem using conventional analyses. Furthermore, conventional analyses tend to be inefficient when used to examine the interactions among many different contributing parameters $[20,21]$. As an alternative solution, machine learning 
can offer an improved means of prediction for a wide range of parameters using fewer simulation runs. Predictor correlations developed via machine learning can be used to solve problems involving a large number of inter-related variables. It is therefore not surprising that artificial intelligence algorithms have been increasingly used in thermal analysis (see Refs. [22, 29] for examples). However, such studies are still rather limited, implying that further efforts are needed to exploit more effectively the broad capabilities of artificial intelligence in thermodynamic analysis.

By combining particle swarm optimization (PSO) and artificial neural networks (ANNs), Rashidi et al. [22] investigated entropy generation in a transient flow across a rotating disc in a magnetic field. Siavashi et al. [23] performed an optimization study to maximize the heat transfer and minimize the pressure drop of a heat exchanger tube filled with multi-layered porous foam and nanofluids. The porosity and nanoparticle concentration were found to have substantial effects on the target parameters. Using a genetic algorithm (GA), Shahsavar et al. [24] optimized the non-Newtonian nanofluid flow in a concentric annulus, with the aim of minimizing irreversibilities and maximizing heat transfer. The geometric parameters and nanoparticle concentration were found to be the most influential parameters. Bahiraei et al. [25] used a multi-layer perception (MLP) neural network to analyze nanofluid-based solar equipment fitted with a thermoelectric module. They found that in comparison with pure MLP, ensemble algorithms, such as GA-MLP and imperialist competition algorithm (ICA)-MLP, can generate improved predictions. Using PSO, Keykhan et al. [26] optimized a porous-volume tube filled with an Ag-water nanofluid. By increasing the porosity or decreasing the nanoparticle concentration, they were able to decrease the Nusselt number and friction factor. The transport characteristics of a reactive flow of hybrid nanofluid in a porous medium were analyzed by Mohebbi Najm Abad et al. [27] and Alizadeh et al. [28]. These researchers trained a neural network so that it could predict the response of a thermal system to changes in the key parameters. PSO was used to develop estimator correlations for improved accuracy. Those studies demonstrated the potential of predictor intelligence algorithms in unravelling complex thermochemical problems. Ebrahimi-Moghadam et al. [29] used an ANN and a quadratic algorithm to minimize entropy generation and identify the optimum volume fraction in a parabolic collector. Introducing nanoparticles to the base fluid was found to decrease thermal entropy generation and increase irreversibilities due to friction.

As mentioned earlier, using conventional computational-fluid-dynamics algorithms to solve complex thermo-hydrodynamic problems can be numerically costly. As an alternative tool, estimator methods are preferred because they are well suited to tackling problems involving many inter-related variables. Leveraging the power of ANNs, the present study aims to uncover new physical insight into the thermodynamics and thermal convection of a hybrid nanofluid flowing around a cylinder in porous media. A number of estimator correlations for the target parameters are developed with the aid of PSO. 


\section{Numerical methodology}

\subsection{Problem description and governing equations}

Fig. 1 shows a diagram of the present flow system. A cylinder of radius $a$ is embedded inside a porous medium. A nanofluid of strain rate $\bar{k}$ flows externally around the surface of the cylinder, producing a stagnation point. The assumptions made in the analysis are:

- The fluid is Newtonian.

- The study assumes a single-phase nanofluid.

- The flow of nanofluid is steady, laminar and incompressible.

- The cylinder is infinite in length with a constant wall temperature.

- The porous medium is in local thermal equilibrium, as well as being homogenous and isotropic.

- Viscous heating, gravitational acceleration, thermal radiation and dissipation are ignored.

- The thermophysical properties are constant.

- The Reynolds number, based on the pore dimension in the porous medium, is low to moderate, implying that nonlinear momentum transfer is negligible.

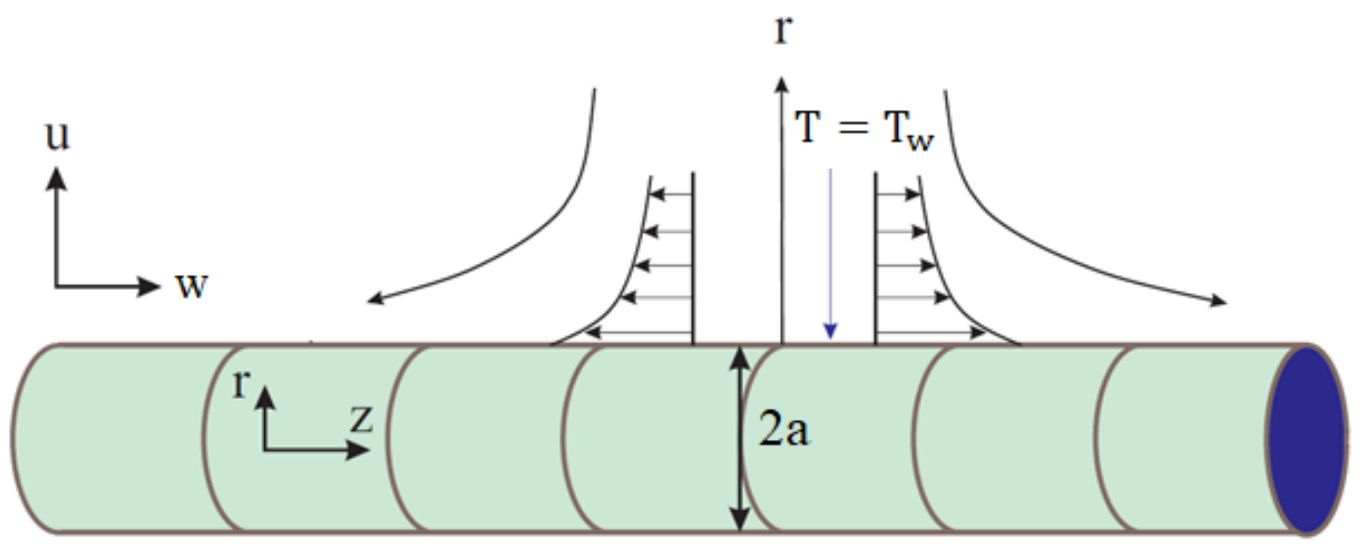

Fig. 1 Diagram of the present flow system.

Numerical simulation of the present flow system involves the two-dimensional Darcy-Brinkman momentum model along with the one-equation energy equation in cylindrical coordinates [30-33]. The governing equations are those for continuity, momentum (radial and axial) and energy, respectively:

$\frac{\partial(r u)}{\partial r}+r \frac{\partial w}{\partial z}=0$

$\frac{\rho_{h n f}}{\varepsilon^{2}}\left(u \frac{\partial u}{\partial r}+w \frac{\partial u}{\partial z}\right)=-\frac{\partial p}{\partial r}+\frac{\mu_{h n f}}{\varepsilon}\left(\frac{\partial^{2} u}{\partial r^{2}}+\frac{1}{r} \frac{\partial u}{\partial r}-\frac{u}{r^{2}}+\frac{\partial^{2} u}{\partial z^{2}}\right)-\frac{\mu_{h n f}}{k_{1}} u$, 
$\frac{\rho_{h n f}}{\varepsilon^{2}}\left(u \frac{\partial w}{\partial r}+w \frac{\partial w}{\partial z}\right)=-\frac{\partial p}{\partial z}+\frac{\mu_{h n f}}{\varepsilon}\left(\frac{\partial^{2} w}{\partial r^{2}}+\frac{1}{r} \frac{\partial w}{\partial r}+\frac{\partial^{2} w}{\partial z^{2}}\right)-\frac{\mu_{h n f}}{k_{1}} w$,

$u \frac{\partial T}{\partial r}+w \frac{\partial T}{\partial z}=\frac{k_{h n f}}{\left(\rho \cdot C_{p}\right)_{h n f}}\left(\frac{\partial^{2} T}{\partial r^{2}}+\frac{1}{r} \frac{\partial T}{\partial r}+\frac{\partial^{2} T}{\partial z^{2}}\right)$.

In Eqs. (1)-(4), $\rho_{h n f}, p, \mu_{h n f}, T, k_{h n f},\left(\rho . C_{p}\right)_{h n f}$, and $k_{1}$ are the density, pressure, dynamic viscosity, temperature, thermal conductivity, heat capacitance and permeability of the porous medium, respectively.

The velocity boundary conditions are:

$r=a: \quad u=0, w=0$,

$r=\infty: \quad u=-\bar{k}\left(r-\frac{a^{2}}{r}\right), \quad w=2 \bar{k} z$.

Hydrodynamic no-slip conditions are applied to the external surface of the cylinder, as per Eq. (5), while the velocity approaches that given by the potential flow assumption at a distance of infinity, as per Eq. (6) [30,31,34].

The thermal boundary conditions are:

$r=a: \quad T=T_{w}=$ constant $=$ temperature of the cylinder surface,

$r=\infty: \quad T=T_{\infty}=$ free-stream temperature,

\subsection{Flow solutions}

Through the following transformation, the governing equations [Eqs. (1)-(4)] are simplified:

$u=-\frac{\bar{k} \cdot a}{\sqrt{\eta}} f(\eta), \quad w=\left[2 \bar{k} f^{\prime}(\eta)\right] z, \quad p=\rho_{f} \bar{k}^{2} a^{2} P$

where $\eta=\left(\frac{r}{a}\right)^{2}$ is the non-dimensional radial variable. Substitution of Eq. (8) into Eq. (1) turns Eqs.

(2) and (3) into a system of coupled ordinary differential equations:

ع. $\left[\eta f^{\prime \prime \prime}+f^{\prime \prime}\right]+A_{1} \cdot A_{2} \cdot \operatorname{Re}\left[1+f f^{\prime}-\left(f^{\prime}\right)^{2}\right]+\varepsilon^{2} \cdot \lambda\left[1-f^{\prime}\right]=0$,

$P-P_{0}=-\frac{A_{2}}{2 \varepsilon^{2}}\left(\frac{f^{2}}{\eta}\right)-\frac{1}{A_{1} \cdot \varepsilon}\left[\left(\frac{f^{\prime}}{R e}\right)+\frac{\lambda}{A_{1} \cdot \operatorname{Re}} \int_{1}^{\eta} \frac{f}{\eta} d \eta\right]-2\left[\frac{A_{2}}{\varepsilon^{2}}+\frac{\lambda}{A_{1} \cdot \operatorname{Re}}\right]\left(\frac{z}{a}\right)^{2}$,

where $R e=\frac{\bar{k} \cdot a^{2}}{2 v_{f}}$ is the Reynolds number (at $T_{\infty}$ ), $\lambda=\frac{a^{2}}{4 k_{1}}$ is the permeability parameter (Definition of permeability parameter is the inverse of Darcy number), and the prime symbol denotes derivatives with respect to $\eta$. Using Eqs. (5), (6) and (8), we transform the boundary conditions for Eqs. (9) and (10) into:

$\eta=1: \quad f^{\prime}(1)=0, \quad f(1)=0$, 
$\eta \rightarrow \infty: \quad f^{\prime}(\infty)=1$

Introducing equation 13 then yields:

$\theta(\eta)=\frac{T(\eta)-T_{\infty}}{T_{w}-T_{\infty}}$

and through Eq. (8), the energy equation [Eq. (4)] becomes the following non-dimensional equation:

$\eta \theta^{\prime \prime}+\theta^{\prime}+\operatorname{Re} \cdot \operatorname{Pr} \cdot \frac{A_{3}}{A_{4}}\left(f \cdot \theta^{\prime}\right)=0$

while the thermal boundary conditions become:

$\eta=1: \quad \theta(1)=1$

$\eta \rightarrow \infty: \quad \theta(\infty)=0$.

Through the use of an implicit iterative finite-difference algorithm [35,36], Eqs. (9), (10) and (14), along with their boundary conditions [Eqs. (11), (12), (15) and (16)], are solved numerically.

\subsection{Hybrid nanofluid}

Here we use a mix of water and nanoparticles of alumina $\left(\mathrm{Al}_{2} \mathrm{O}_{3}\right)$ and copper $(\mathrm{Cu})$ as our hybrid nanofluid. The $\mathrm{Cu}$ nanoparticles are added to a homogenous mixture of water and $\mathrm{Al}_{2} \mathrm{O}_{3}$. When the $\mathrm{Cu}$ volume fraction $\left(\phi_{2}\right)$ is varied, the $\mathrm{Al}_{2} \mathrm{O}_{3}$ volume fraction $\left(\phi_{1}\right)$ is held constant, and vice versa. The thermophysical properties of this hybrid nanofluid are listed in Table 1.

Table 1. Mathematical formulae of the thermophysical properties of the $\mathrm{Cu}-\mathrm{Al}_{2} \mathrm{O}_{3}$-water hybrid nanofluid [37].

\begin{tabular}{|c|c|}
\hline $\begin{array}{c}\text { Thermophysical } \\
\text { properties }\end{array}$ & Mathematical formulae \\
\hline Fluid density & $\begin{array}{c}\rho_{h n f}=\rho_{f}\left(1-\phi_{2}\right)\left[\left(1-\phi_{1}\right)+\phi_{1}\left(\frac{\rho_{s_{1}}}{\rho_{f}}\right)\right] \\
+\phi_{2} \rho_{s_{2}}\end{array}$ \\
\hline Heat capacity & $\left(\rho . C_{p}\right)_{h n f}=\left(\rho . C_{p}\right)_{f}\left(1-\phi_{2}\right)\left[\left(1-\phi_{1}\right)\right.$ \\
& $\left.+\phi_{1} \frac{\left(\rho \cdot C_{p}\right)_{s_{1}}}{\left(\rho \cdot C_{p}\right)_{f}}\right]$ \\
& $+\phi_{2}\left(\rho \cdot C_{p}\right)_{s_{2}}$ \\
\hline Viscosity & $\mu_{h n f}=\frac{\mu_{f}}{\left(1-\phi_{1}\right)^{2.5}\left(1-\phi_{2}\right)^{2.5}}$ \\
\hline
\end{tabular}




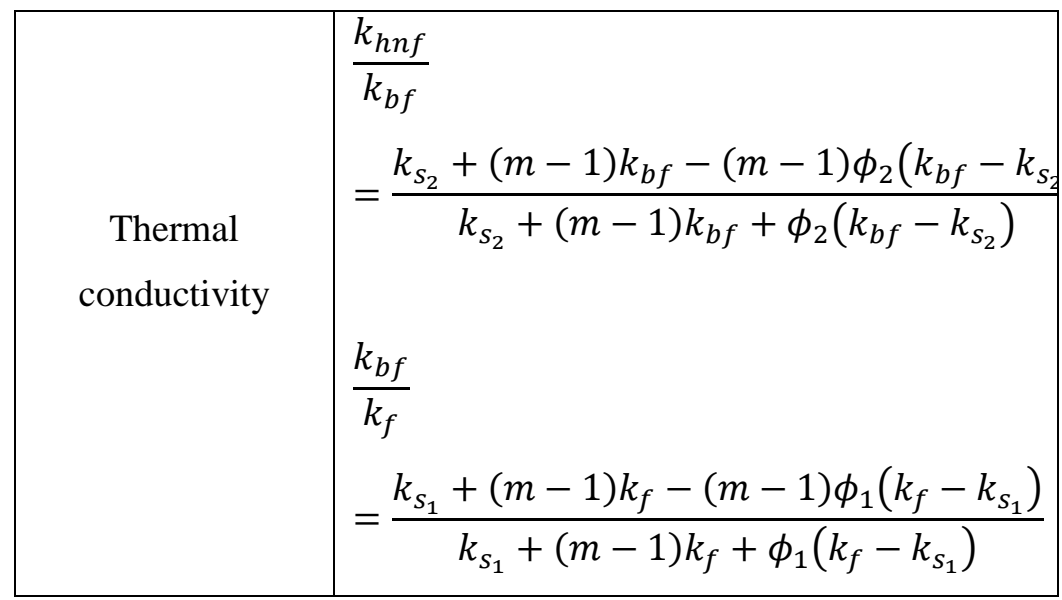

In Table 1, $m$ is the shape factor, which is set to 3 for spherical particles and 5.7 for platelet-shaped particles. Table 2 lists the thermophysical properties of the individual components of the nanofluid at $25^{\circ} \mathrm{C}$ : the base fluid (water) and the constituent nanoparticles $\left(\mathrm{Al}_{2} \mathrm{O}_{3}\right.$ and $\left.\mathrm{Cu}\right)$.

Table 2. Thermophysical properties of the individual components of the $\mathrm{Al}_{2} \mathrm{O}_{3}-\mathrm{Cu}$-water hybrid nanofluid at $25^{\circ} \mathrm{C}[38]$.

\begin{tabular}{|c|c|c|c|}
\hline $\begin{array}{c}\text { Thermophysical } \\
\text { properties }\end{array}$ & $\mathbf{H}_{\mathbf{2}} \mathbf{O}$ & $\mathbf{A l}_{\mathbf{2}} \mathbf{O}_{\mathbf{3}}$ & $\mathbf{C u}$ \\
\hline$\rho\left(\frac{\mathrm{kg}}{\mathrm{m}^{3}}\right)$ & 997.0 & 3970 & 8933 \\
\hline$C_{p}\left(\frac{\mathrm{J}}{\mathrm{kg} \cdot \mathrm{K}}\right)$ & 4180 & 765 & 385 \\
\hline$k\left(\frac{\mathrm{W}}{\mathrm{m} \cdot \mathrm{K}}\right)$ & 0.6071 & 40 & 400 \\
\hline
\end{tabular}

\section{Numerical parameters}

\subsection{Nusselt number and non-dimensional shear stress}

The cylinder wall experiences a shear stress due to the hybrid nanofluid flow, which is given by [32,39]:

$$
\sigma=\mu_{h n f}\left[\frac{\partial w}{\partial r}\right]_{r=a},
$$

where $\mu_{h n f}$ is the dynamic viscosity of the hybrid nanofluid. Eq. (12) is used to obtain a semi-similar solution for the shear stress:

$\sigma=\mu_{h n f} \frac{2}{a}\left[2 \bar{k} z f^{\prime \prime}(1)\right] \Rightarrow \frac{\sigma a}{4 \mu_{f} \bar{k} z}=\frac{1}{A_{1}} f^{\prime \prime}(1)$.

The local coefficient of heat transfer on the external cylinder wall is defined as: 
$h=\frac{q_{w}}{T_{w}-T_{\infty}}=\frac{-k_{h n f}\left(\frac{\partial T}{\partial r}\right)_{r=a}}{T_{w}-T_{\infty}}=-\frac{2 k_{h n f}}{a} \frac{\partial \theta(1)}{\partial \eta}$,

where the heat flux $q_{w}$ is given by:

$q_{w}=-\frac{2 k_{h n f}}{a} \frac{\partial \theta(1)}{\partial \eta}\left(T_{w}-T_{\infty}\right)$.

Consequently, the Nusselt number can be expressed as:

$N u=\frac{h a}{2 k_{f}}=-\frac{k_{h n f}}{k_{f}} \theta^{\prime}(1)=-A_{4} \cdot \theta^{\prime}(1)$.

\subsection{Entropy generation}

The entropy generated in the present flow system is computed via the following [40,41]:

$$
\begin{aligned}
\dot{S}^{\prime \prime \prime} \text { gen }=\frac{k_{h n f}}{T_{W}^{2}}\left(\frac{\partial T}{\partial r}\right)^{2}+ & \frac{2 \mu_{h n f}}{T_{W}}\left[\left(\frac{\partial u}{\partial r}\right)^{2}+\left(\frac{u}{r}\right)^{2}+\left(\frac{\partial w}{\partial z}\right)^{2}+\frac{1}{2}\left(\frac{\partial w}{\partial r}\right)^{2}\right]+ \\
& +\frac{\mu_{h n f}}{k_{1} \cdot T_{W}}\left[u^{2}+w^{2}\right] .
\end{aligned}
$$

Substituting the similarity variables from Eq. (12) into Eq. (22), we simplify the expression for the local generation of entropy to:

$$
\begin{gathered}
\dot{S}^{\prime \prime \prime}{ }_{\text {gen }}=\frac{4 k_{h n f}\left(T_{W}-T_{\infty}\right)^{2}}{a^{2} T_{W}^{2}}\left[\eta \theta^{\prime 2}\right] \\
+\frac{4 \bar{k}^{2} \mu_{h n f}}{T_{W}}\left[\eta f^{\prime \prime 2}+4 f^{\prime 2}+\frac{f^{2}}{\eta^{2}}-\frac{2 f f^{\prime}}{\eta}\right]+\frac{\bar{k}^{2} \mu_{h n f} a^{2}}{k_{1} T_{W}}\left[\left(\frac{f}{\eta}\right)^{2}+4{f^{\prime}}^{2}\right],
\end{gathered}
$$

where $N_{G}=\frac{\dot{S}^{\prime \prime \prime} \text { gen }}{S^{\prime \prime \prime}{ }_{0}}$ and $S^{\prime \prime \prime}{ }_{0}=\frac{4 k_{f}\left(T_{W}-T_{\infty}\right)}{a^{2} T_{W}}$ is the characteristic rate of entropy generation.

By incorporating the similarity variables from the local volumetric rate of entropy generation, we find $N_{G}$ via:

$N_{G}=\Lambda \cdot A_{4}\left[\eta \theta^{\prime 2}\right]$
$+\frac{B r}{A_{1}}\left\{\left[\eta f^{\prime \prime 2}+4{f^{\prime}}^{2}+\frac{f^{2}}{\eta^{2}}-\frac{2 f f^{\prime}}{\eta}\right]+\lambda\left[\left(\frac{f}{\eta}\right)^{2}+4 f^{\prime 2}\right]\right\}$,

where $\Lambda=\frac{\left(T_{W}-T_{\infty}\right)}{T_{W}}$ is the non-dimensional temperature difference, and $B r=\frac{\mu_{f}(\bar{k} \cdot a)^{2}}{k_{f}\left(T_{W}-T_{\infty}\right)}$ is the Brinkman number. Meanwhile, the Bejan number $\left(\frac{\text { Thermal source value }}{\text { Total entropy generation }}\right)$, is used here to gain physical insight into the contributions of the different thermal mechanisms to the total energy loss. This metric is defined here as:

$$
B e=\frac{\Lambda \cdot A_{4}\left[\eta{\theta^{\prime}}^{2}\right]}{\frac{B r}{A_{1}}\left\{\left[\eta f^{\prime \prime 2}+4 f^{\prime 2}+\frac{f^{2}}{\eta^{2}}-\frac{2 f f^{\prime}}{\eta}\right]+\lambda\left[\left(\frac{f}{\eta}\right)^{2}+4{f^{\prime}}^{2}\right]\right\}} .
$$




\section{Grid independence and validation}

To test for grid independence, we compare five different meshes (Fig. 2): $51 \times 18,102 \times 36$, $204 \times 72,408 \times 144$ and $816 \times 288$ cells, corresponding to $\eta$ and $\varphi$. Fig. 2 shows that the $408 \times$ 144 mesh achieves grid independence. For all the governing equations, the convergence criteria, defined as the difference between two successive residuals, are set to $10^{-7}$. Discretization of the governing equations to algebraic form is performed to second-order numerical accuracy $[35,42]$.

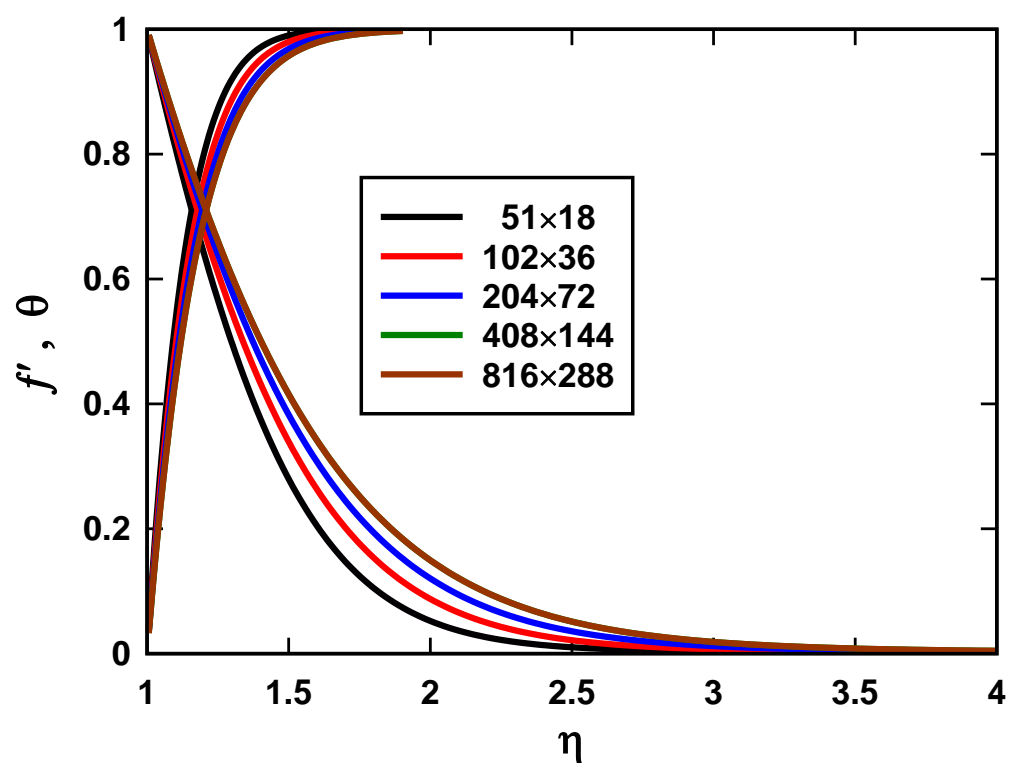

Fig. 2 Grid independence test at $R e=10, \lambda=10, \lambda_{1}=1.0, M=1.0, B i=0.1, R_{d}=$

$$
1.0, \theta_{w}=1.2
$$

To validate this numerical framework, we compare the present results against previous work. In particular, the axial velocity and temperature on the external surface of the cylinder are inspected for comparison, with the cylinder removed from the porous layer (large permeability). Fig 3 shows that there is close agreement between the two sets of axial velocity and temperature data. 
(a)

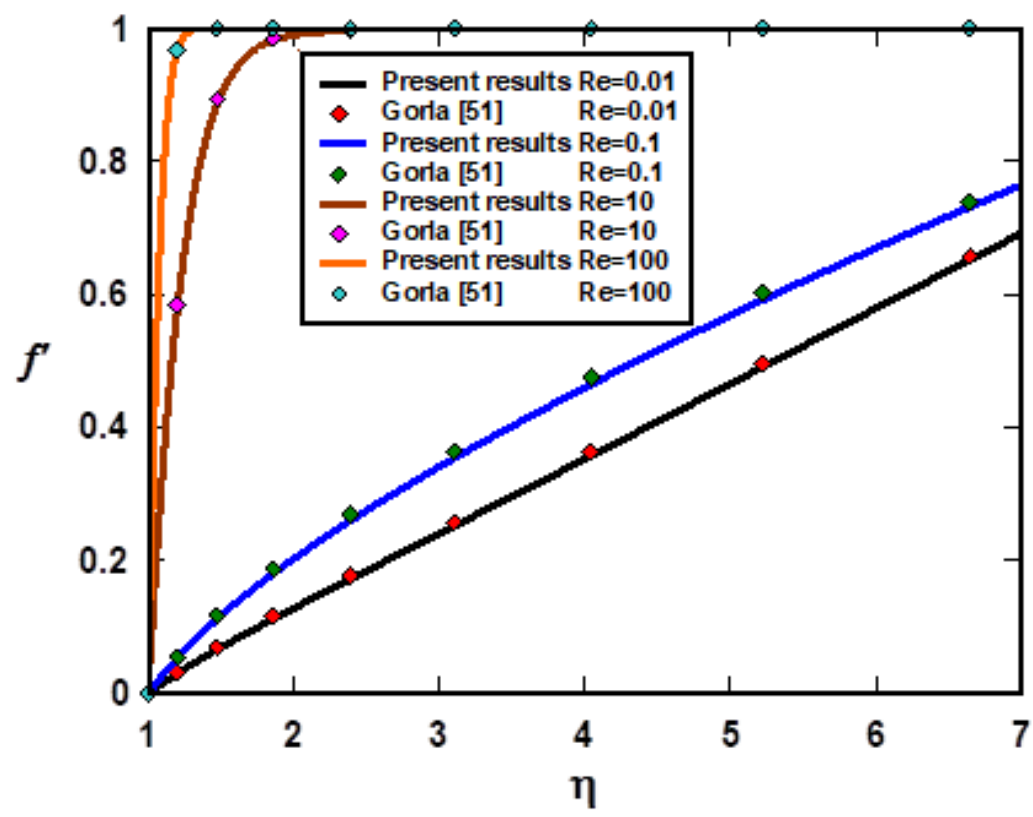

(b)

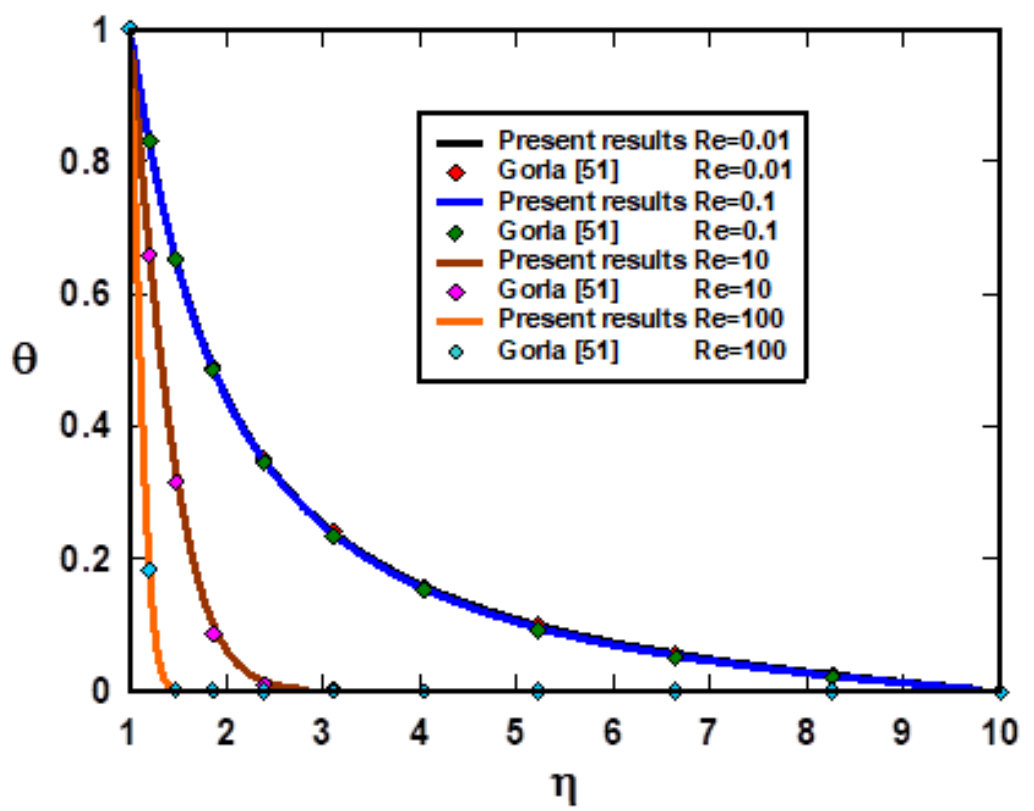

Fig. 3 Comparison of the present results against those of Gorla [51] at different values of $R e$.

\section{Estimator and optimization algorithms}

\section{1. Feed-forward artificial neural network}

Originally inspired by biological neural networks [46], an ANN is a versatile tool for optimization and estimation. An ANN needs to learn (or be trained) to perform specific tasks, but it does not usually require task-specific rules to be defined. It consists of a series of artificial neurons, acting analogously to biological neurons [47]. In the present study, a MLP network is used to estimate selected parameters. The network has three primary layers: (i) an input layer, (ii) a hidden layer, and 
(iii) an output layer. Input signals are first transmitted to the neurons (shown as circles in Fig. 4), which are linked by connections (lines) that are adjustable via a weight factor.

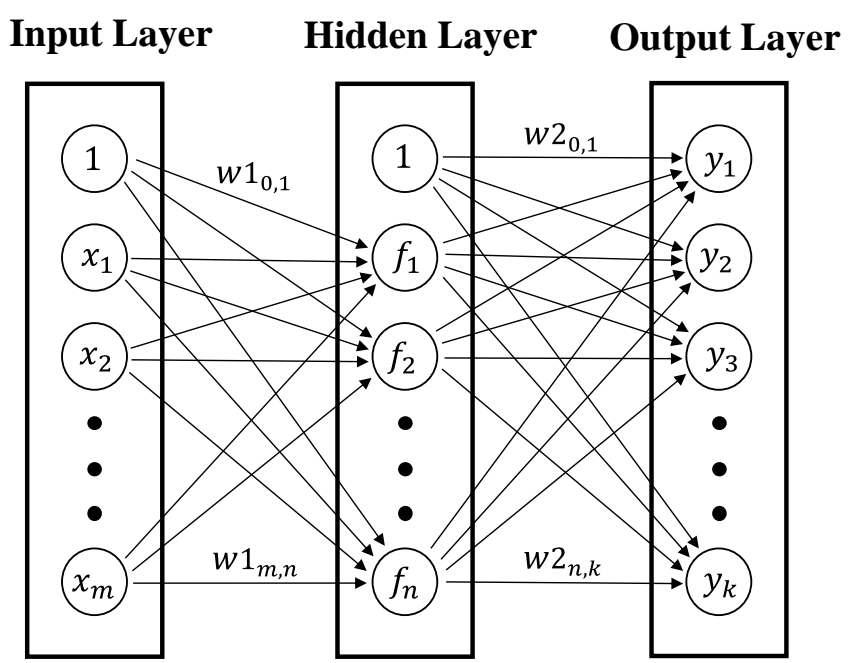

Fig. 4. Graphic representation of a typical ANN with three layers.

The input layer contains $n+1$ neurons for $n$ input(s). All neurons receive input data, except the first neuron, which receives an assigned value of 1 . In the hidden layer, the total number of neurons is $m+1$. During the construction of a network, the correct value of $m$ is evaluated by running a series of tests. In the hidden layer, there is one neuron that plays a bias role, with the other neurons subjected to an activation function. The sum of the weighted outputs for the output layer is given by [47]:

$$
f_{i}=g\left(w_{0, i}+\sum_{j=1}^{n} w_{j, i} x_{j}\right)
$$

where $w_{0, i}$ is the weight applied to the $i$-th neuron in the hidden layer. Meanwhile, $w_{j, i}$ represents the weight factor applied to the $j$-th input of the $i$-th neuron in the hidden layer. Moreover, $x_{j}$ and $f_{i}$ respectively represent the $j$-th input of the ANN as well as the output of the $i$-th neuron. The activation function $g$ can be exponential, logistic or hyperbolic.

The neurons of the output layer further operate on the network outputs. This is similar to the processes in the hidden layer and can be expressed as [47]:

$$
\text { Out }_{i}=h\left(v_{0, i}+\sum_{j=1}^{m} v_{j, i} f_{j}\right),
$$


where $v_{0, i}$ denotes the weights applied to the bias and the $j$-th input in the hidden layer. Furthermore, $v_{j, i}$ is the $i$-th neuron of the output layer.

In the present study, an error propagation algorithm is used for ANN training. Such a process trains the weights applied to the ANN layers via a supervised learning procedure. The difference between the ANN outputs and the anticipated outputs within the learning dataset is used to calculate the learning error rate. It is worth noting that the configuration of the MLP network can have major effects on its performance. In the interest of a lower error rate, the optimal configuration of the network is found by repeating tests with different choices. Thus, the activation function used for the neurons in the hidden and output layers, as well as the number of neurons in the hidden layer, are found via iteration. In this process, we consider different parameter values and compute the errors of the model.

\subsection{Selecting features via mutual information}

Features are selected by maximizing a criterion for the outputs of the model and by minimizing the mutual information (MI) among all the considered features. Here, the MI is calculated as:

$$
I(x ; y)=\iint p(x, y) \log \frac{p(x)}{p(x) p(y)} d x d y
$$

where $p(x), p(y)$ and $p(x, y)$ are the probability density functions of $x, y$, and their mutual occurrence, respectively.

To reduce the model error, the maximum dependency on the model outputs is required. To this end, the minimum-redundancy-maximum-relevance (MRMR) algorithm is used to identify a group of features with the strongest correlations among the model outputs and with the weakest correlations among the model inputs. The temporal dependence of MRMR is exponential, implying that mathematical operations are needed to retrieve a large number of parameters. To achieve this, we use the mutual information difference (MID) and the F-test correlation difference (FCD) [48]. The latter is computed as follows [49]:

$$
M I D=\max _{i \in \varphi(S)}\left[I(i, h)-\frac{1}{\left|S_{f}\right|} \sum_{j \in S} I(i, j)\right],
$$

where $S_{f}$ is a group of selected features.

\subsection{Particle swarm optimization (PSO)}


In PSO, random solutions are first collected, then updates are generated, and finally an optimal solution is sought [50]. During each iteration, the two best values are fed to the particles. The best solution obtained by a particle during its motion represents the first of these and is known as the pbest value. The other 'best' refers to the value obtained by all the particles, known as the gbest value.

To find the best solution, it is assumed that the particles enter the search space. The following equation is used [52]:

$$
v_{i}(t+1)=w v_{i}(t)+c_{1} r_{1}\left[\operatorname{pbest}(t)-x_{i}(t)\right]+c_{2} r_{2}\left[\operatorname{gbest}(t)-x_{i}(t)\right],
$$

where $t$ and $i$ are the iteration and index numbers, respectively. Furthermore, $x$ and $v$ are the location and velocity of the particle, and $r_{1}$ and $r_{2}$ are the random values produced each time the equation is implemented. Meanwhile $c_{1}$ and $c_{2}$ are acceleration coefficients, whose values are usually between zero and two [53]. Once the velocity of a particle is found, its position can be calculated via the following equation [52]:

$$
x_{i}(t+1)=x_{i}(t)+v_{i}(t+1) .
$$

\section{Results and discussion}

Simulations are performed to generate the data required for ANN training. Several neural intelligence models - such as MLP, radial basis function (RBF), support vector regression (SVR) [43] and least mean squares (LMS) [44] - are compared so as to identify the optimal method. Fig. 5 shows this comparison in terms of the entropy generated. Although similar procedures are performed for the other parameters (i.e. the Nusselt and Bejan numbers), they are not shown here as they are in qualitative agreement with Fig. 5. This figure confirms the more accurate results of the MLP method in comparison with the other algorithms, through an evaluation of the mean absolute error (MAE). Thus, the MLP method is used here. Fig. 5 shows that the MLP method exhibits progressively better performance as the number of features grows. The full ordering of the feature prioritization for entropy generation, the Nusselt number, and the Bejan number are listed in Tables 3, 4 and 5, respectively. 


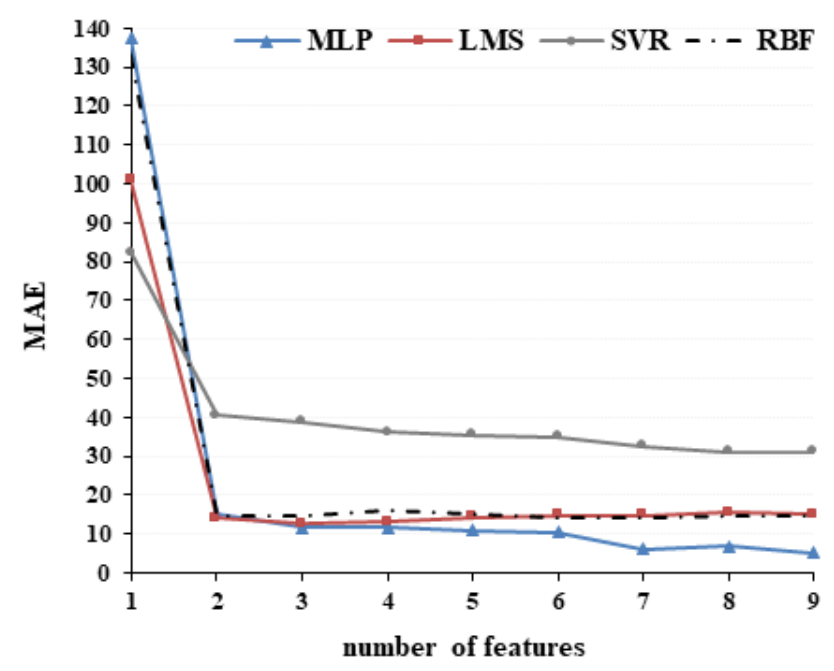

Fig. 5. Mean absolute error (MAE) in estimating the entropy generation with various potential models.

Table 3. Order of prioritization for features related to the entropy generation number.

\begin{tabular}{|c|c|c|c|c|c|c|c|c|c|}
\hline Ordering & 1 & 2 & 3 & 4 & 5 & 6 & 7 & 8 & 9 \\
\hline Feature & $B r$ & $\lambda$ & $\eta$ & $R e$ & $P r$ & $\phi_{1}$ & $\phi_{2}$ & $M$ & $D$ \\
\hline
\end{tabular}

Table 4. Order prioritization for features related to the Nusselt number.

\begin{tabular}{|c|c|c|c|c|c|c|}
\hline Ordering & 1 & 2 & 3 & 4 & 5 & 6 \\
\hline Feature & $\phi_{2}$ & $R e$ & $\phi_{1}$ & $\operatorname{Pr}$ & $M$ & $\lambda$ \\
\hline
\end{tabular}

Table 5. Order of prioritization for features related to the Bejan number.

\begin{tabular}{|c|c|c|c|c|c|c|c|c|c|}
\hline Ordering & 1 & 2 & 3 & 4 & 5 & 6 & 7 & 8 & 9 \\
\hline Feature & $\eta$ & $D$ & $\operatorname{Pr}$ & $\lambda$ & $\operatorname{Re}$ & $B r$ & $M$ & $\phi_{1}$ & $\phi_{2}$ \\
\hline
\end{tabular}

The default values of the parameters used in Figs. 6-10 are listed in Table 6. Fig. 6 shows predictions of the temperature in non-dimensional form. It can be seen that increasing the shape factor (i.e. platelet-shaped nanoparticles) improves heat transfer and reduces the non-dimensional temperature. As the permeability parameter increases, the non-dimensional temperature begins to saturate (Fig. 6a). Thus, reducing the permeability of the porous medium enhances the heat transfer from the cylinder. It is worth noting that similar findings have been reported before for simpler flows moving through porous media [2,3]. Furthermore, as the volume fraction of nanoparticles increases, so too does the difference between the local temperature and its value at infinity. This is because the thermal boundary layer becomes thicker owing to the higher thermal conductivity of the nanofluid arising 
from the higher nanoparticle concentration [31]. In turn, this increases the non-dimensional temperature, as discussed in Ref. [21]. Fig. 6b shows that this behavior can be reproduced by increasing the concentration of either one of the two nanoparticle components. Moreover, Fig. 6b shows that increasing the Reynolds number causes the non-dimensional temperature to drop. This is attributed to the enhanced forced convection at higher Reynolds numbers, a phenomenon that has been reported in previous studies [21,30].

Table 6. Default numerical values of the parameters in Figs. 6-10.

\begin{tabular}{|c|c|c|c|c|c|c|c|c|c|c|c|c|c|}
\hline Parameter & $R e$ & $\lambda_{1}$ & $\phi_{2}$ & $\operatorname{Pr}$ & $m$ & $\phi_{1}$ & $\Lambda$ & $\lambda$ & $K$ & $B i$ & $B r$ & $\lambda$ & $S c$ \\
\hline Numerical values & 10.0 & 10.0 & 0.05 & 1.0 & 3 & 0.05 & 1 & 100 & 1.0 & 0.1 & 1 & 1.5 & 0.1 \\
\hline
\end{tabular}

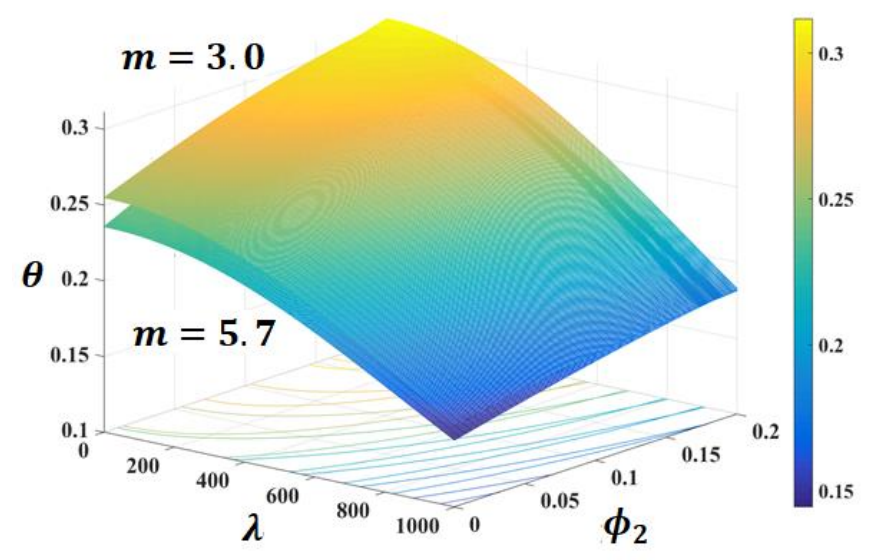

(a)

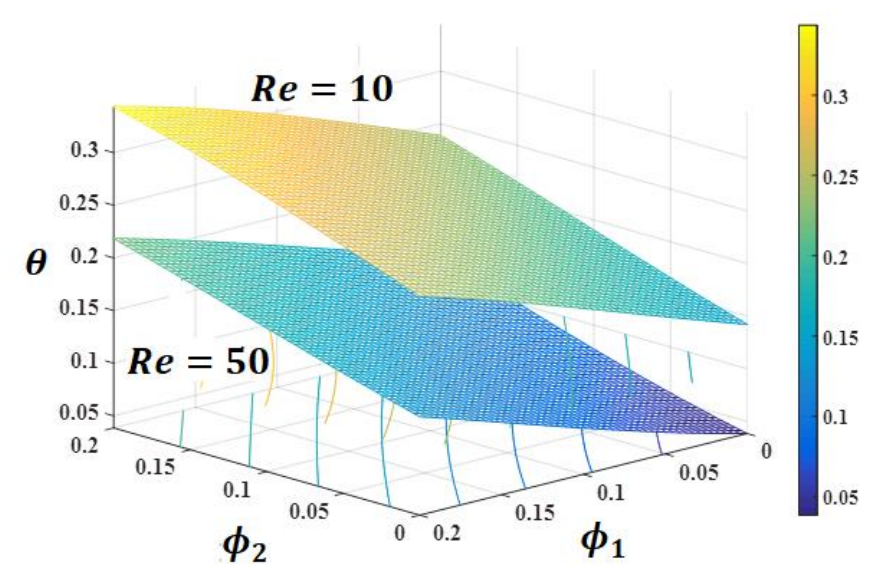

(b)

Fig. 6. Non-dimensional temperature $(\theta)$ response to (a) the shape factor of nanoparticles and (b) the

Reynolds number $(R e)$. Data are shown for several values of the permeability parameter $(\lambda)$, the volume fraction of $\mathrm{Al}_{2} \mathrm{O}_{3}$ nanoparticles $\left(\phi_{1}\right)$, and that of $\mathrm{Cu}$ nanoparticles $\left(\phi_{2}\right)$. 
Fig. 7 shows the non-dimensional entropy generation $\left(N_{G}\right)$ as several key parameters are varied. Smaller Brinkman numbers indicate smaller fluid viscosities and larger thermal conductivities. Both of these effects tend to reduce irreversibilities. At smaller Brinkman numbers, the fluid conductivity is high, so adding nanoparticles has no considerable effect on the generation of entropy. Nonetheless, increasing the Brinkman number causes the flow friction to increase, contributing to greater irreversibilities. Fig. 7a shows a single-maximum response surface, indicating a complex contribution of the nanoparticle concentration to the thermal and frictional behavior of the system. Reducing the Reynolds number is seen to suppress convective heat transfer, strengthening gradients in flow temperature and contributing to greater irreversibilities, as shown in Fig. 7b. Furthermore, reducing the permeability parameter promotes entropy generation by impeding flow through the porous medium [37]. However, it also impairs heat transfer [31], amplifying temperature gradients and generating more entropy.

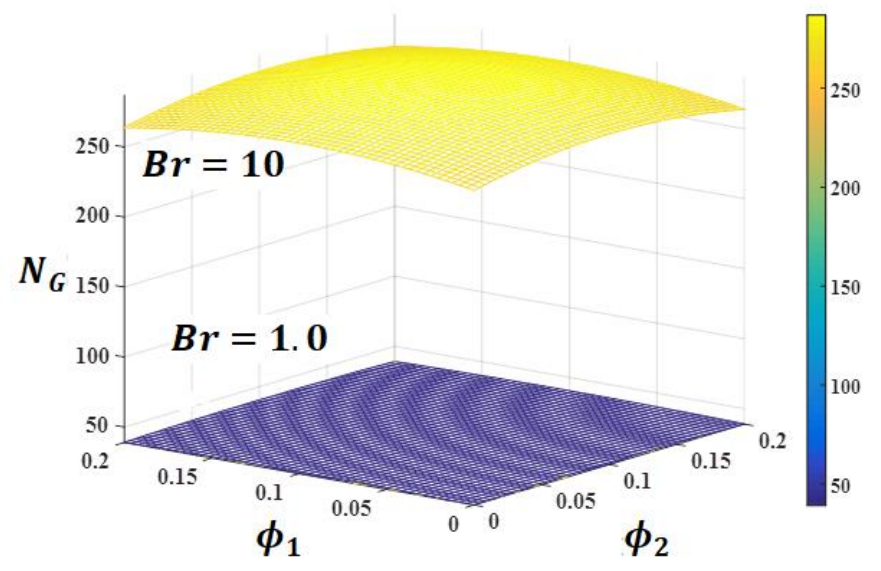

(a)

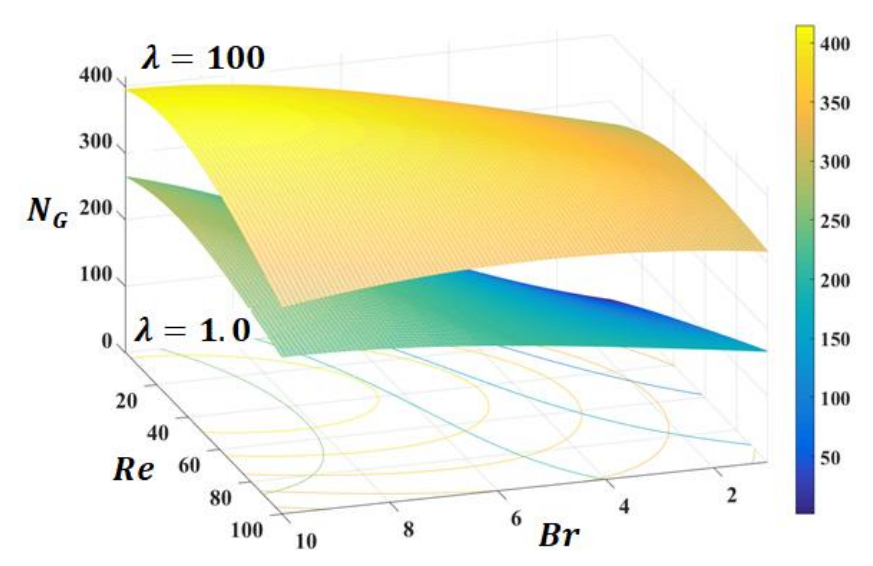

(b)

Fig. 7. Non-dimensional entropy generation $\left(N_{G}\right)$ response to (a) the Brinkman number $(B r)$ and (b) the permeability parameter $(\lambda)$. Data are shown for several values of the Reynolds number $(R e)$, the volume fraction of $\mathrm{Al}_{2} \mathrm{O}_{3}$ nanoparticles $\left(\phi_{1}\right)$, and that of $\mathrm{Cu}$ nanoparticles $\left(\phi_{2}\right)$. 
Fig. 8 shows the variation in the Bejan number $\left(\frac{\text { Thermal entropy generation }}{\text { Total entropy generation }}\right)$. From Fig. 8a, it can be seen that increasing the shape factor of nanoparticles enhances heat transfer, thus weakening thermal gradients and impairing entropy generation. Although increasing the permeability parameter increases entropy generation, it has no significant effect on the Bejan number, owing to greater frictional irreversibilities and greater total entropy generation. Increasing the Prandtl number decreases thermal diffusivity and hence thermal convection, but it also increases thermal entropy generation, giving rise to higher Bejan numbers. As Fig. 8b shows, increasing the Reynolds number also increases the Bejan number. Fig. 7 already showed that increasing the Brinkman number weakens temperature gradients and impairs the generation of entropy.

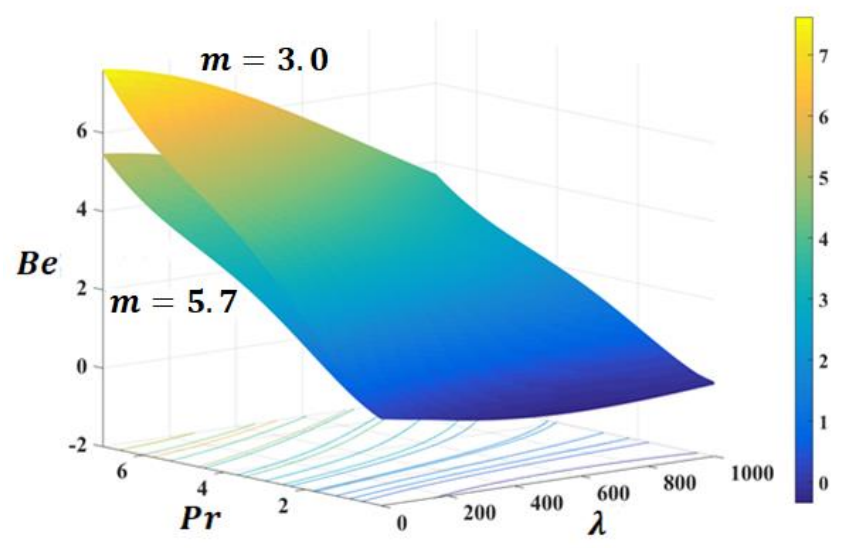

(a)

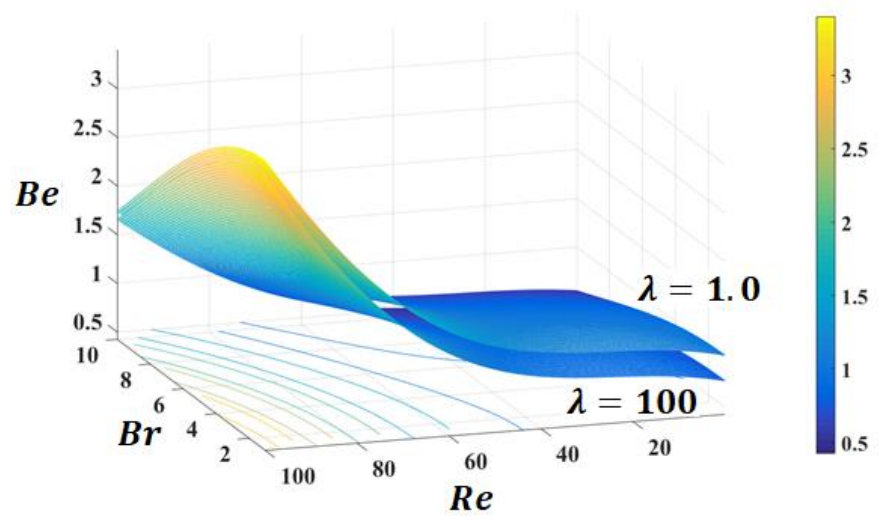

(b) 


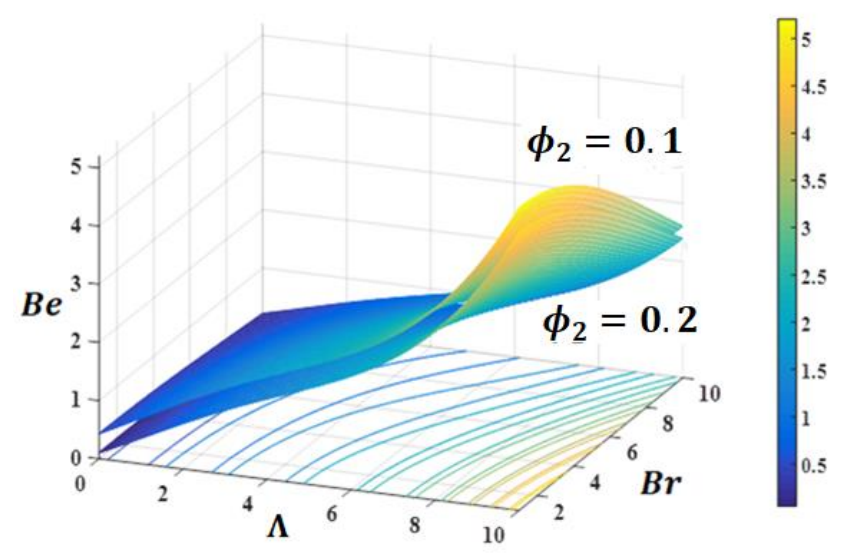

(c)

Fig. 8. Bejan number $(\mathrm{Be})$ response to (a) the shape factor of nanoparticles, (b) the permeability parameter $(\lambda)$ and (c) the volume fraction of $\mathrm{Cu}$ nanoparticles $\left(\phi_{2}\right)$. Data are shown for several values of the Prandtl number $(P r)$, Brinkman number $(B r)$, Reynolds number $(R e)$, and the ratio of the temperature at the cylinder wall to that at infinity $(\Lambda)$.

It will be shown later that increases in the concentration of nanoparticles can enhance the heat transfer rate, thus suppressing thermal entropy generation. This explains the trend seen in Fig. $8 \mathrm{c}$, in which the Bejan number drops at higher values of the nanoparticle volume fraction. Increasing $\Lambda-$ the ratio of the temperature at the wall to that at infinity - increases the thermal gradient and hence the Bejan number.

Fig. 9 shows the non-dimensional shear stress as a function of various parameters. The shear stress is an important consideration in engineering analyses because it affects pumping losses. It can be seen that the non-dimensional shear stress grows as the Reynolds number rises. This occurs because, near the surface of the cylinder, the velocity gradient steepens as the Reynolds number increases. The velocity gradient also steepens as the permeability parameter increases, which can be attributed to a greater resistance to fluid motion. However, the opposite trend is observed when the nanoparticle concentration increases. This could be due to the direct effect of the nanoparticles on the fluid viscosity, which appears in the denominator of the non-dimensional shear stress and therefore acts to reduce it. 


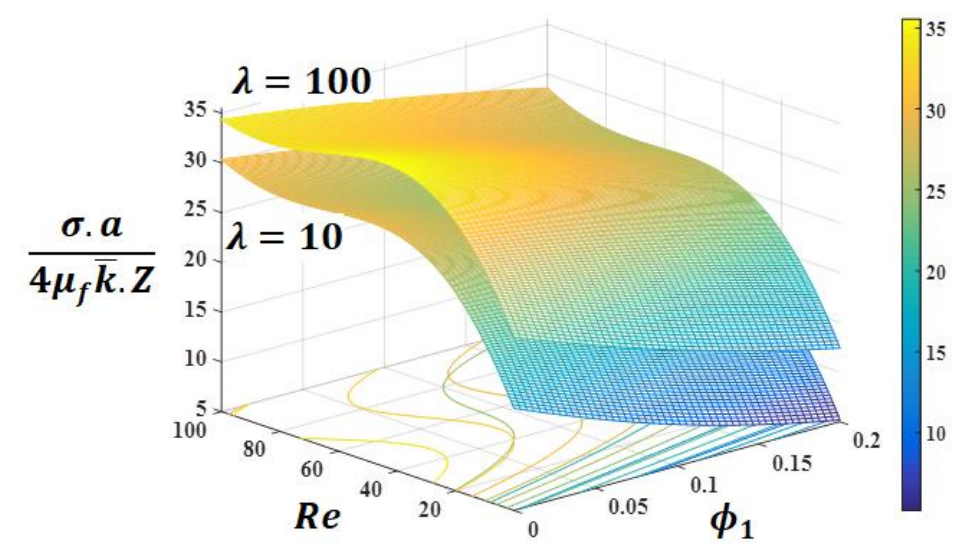

(a)

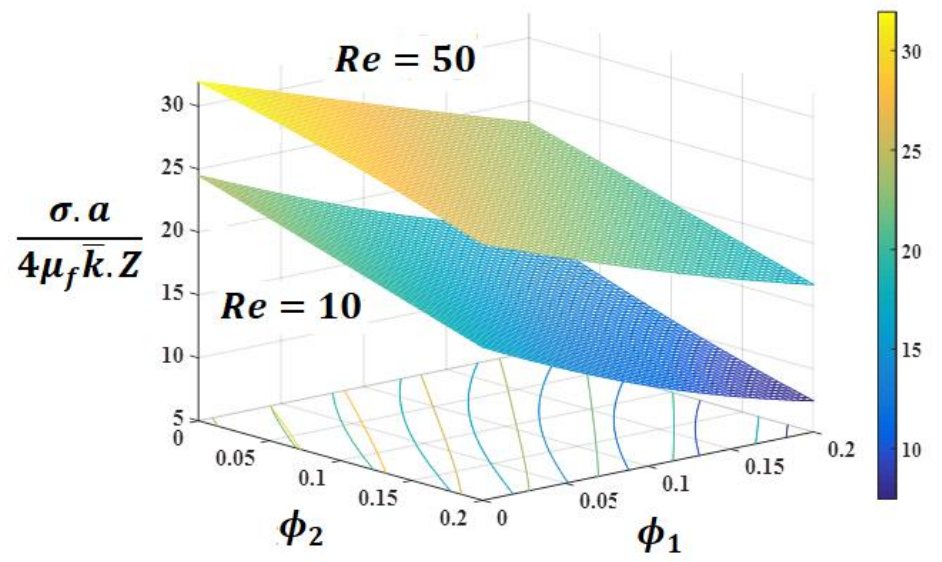

(b)

Fig. 9. Non-dimensional shear stress $\left(\frac{\sigma \cdot a}{4 \mu_{f} \bar{k} \cdot z}\right)$ response to (a) the permeability parameter $(\lambda)$ and (b) the Reynolds number $(R e)$. Data are shown for several values of the volume fraction of $\mathrm{Al}_{2} \mathrm{O}_{3}$ nanoparticles $\left(\phi_{1}\right)$ and that of $\mathrm{Cu}$ nanoparticles $\left(\phi_{2}\right)$.

Figure 10a shows that, consistent with the literature [6-8], the Nusselt number increases as the nanoparticle concentration increases. This trend is further demonstrated in Fig. 10b. As expected from classic heat transfer research, Fig. 10a shows a direct correlation between the Nusselt number and the Reynolds number. However, the dependence of the Nusselt number on the Prandtl number is weaker (Figs. 10a and 10b). Furthermore, the dependence of the Nusselt number on the permeability parameter is particularly strong at low values of this parameter (Fig. 10b). 


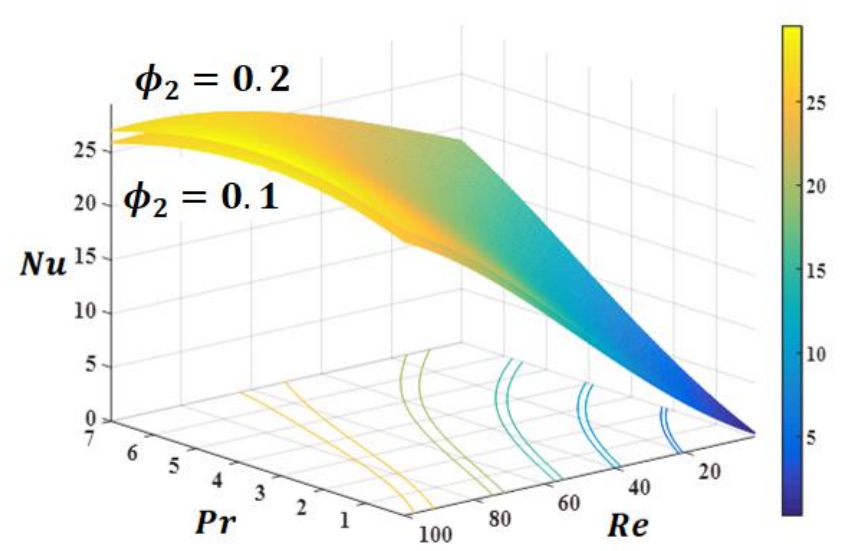

(a)

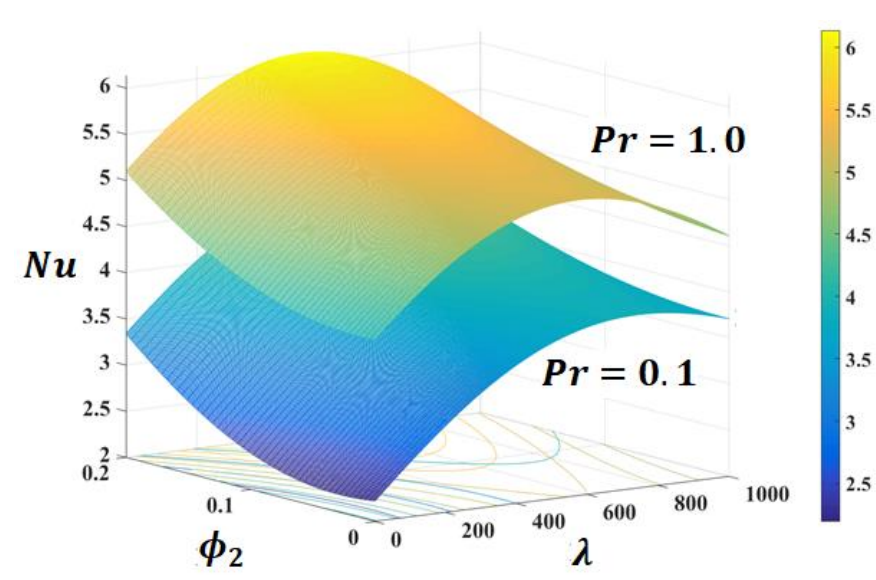

(b)

Fig. 10. Nusselt number ( $N u)$ response to (a) $\phi_{2}$ and (b) the Prandtl number $(P r)$. Data are shown for several values of the Reynolds number $(R e)$ and the permeability parameter $(\lambda)$.

Returning to Fig. 6, it was shown that the temperature discrepancy can be reduced by increasing the permeability parameter, which enhances heat transfer, causing the Nusselt number to rise.

The results presented above have demonstrated the complex behavior of this flow system from thermal, thermodynamic and hydraulic viewpoints. For practical engineering analysis, it is often useful to know the mathematical correlations relating the system characteristics to various control parameters. The predicted results are now used to develop such correlations. It should be noted, however, that the accuracy of the method used to extract the correlations should be within acceptable limits. This requirement can be met by using an optimization algorithm with a high level of accuracy and affordability. Here a PSO algorithm is used to develop heat transfer and hydraulic correlations. The range of parameters used in the correlations are listed in Table 7. The correlations for the Nusselt number are listed in Table 8, along with the MAE. The MAE drops as the number of parameters in the correlations increases. For the most global correlation (with six effective parameters), the MAE is less than 0.5 , which is considered acceptable in this study. Table 9 lists the correlations for the nondimensional shear stress. The effective parameters chosen for this are found via importance ordering 
and by considering the MAE. As well as being an engineering design tool, these correlations can be analyzed to gain fundamental insight into the underlying flow physics. For example, the numerical values of the individual exponents in the correlations represent their relative influences on the nondimensional shear stress and the Nusselt number.

Table 7. Ranges of the parameters used in the correlation extracting procedure.

\begin{tabular}{|c|c|c|c|c|c|}
\hline $0 \leq \phi_{2} \leq 0.4$ & $0.1 \leq R e \leq 100$ & $0 \leq \phi_{1} \leq 0.4$ & $0.1 \leq \operatorname{Pr} \leq 7.0$ & $3 \leq M \leq 5.7$ & $0.1 \leq \lambda \leq 1000$ \\
\hline
\end{tabular}

Table 8. Correlations for the Nusselt number.

\begin{tabular}{|c|c|c|}
\hline Correlation for the Nusselt number & Parameters & $\begin{array}{c}\text { Mean } \\
\text { absolute } \\
\text { error }\end{array}$ \\
\hline$N u=4.099+12.015 \times \phi_{2}^{1.383}$ & $\phi_{2}$ & 1.8293 \\
\hline$N u=2.258+0.800 \times \phi_{2}^{0.574} \times R e^{1.040}$ & $\phi_{2}, R e$ & 1.0218 \\
\hline$N u=3.782+3.083 \times \phi_{2}{ }^{1.048} \times R e^{1.471} \times \phi_{1}^{1.001}$ & $\phi_{2}, R e, \phi_{1}$ & 0.9962 \\
\hline$N u=4.343+11.082 \times \phi_{2}{ }^{1.870} \times R e^{1.971} \times \phi_{1}^{1.742} \times \operatorname{Pr}^{2.124}$ & $\phi_{2}, \operatorname{Re}, \phi_{1}, \operatorname{Pr}$ & 0.5249 \\
\hline$N u=4.277+6.967 \times \phi_{2}{ }^{1.663} \times R e^{1.869} \times \phi_{1}^{1.586} \times \operatorname{Pr}^{2.006} \times M^{0.074}$ & $\phi_{2}, \operatorname{Re}, \phi_{1}, \operatorname{Pr}, M$ & 0.4932 \\
\hline $\begin{array}{c}N u=3.988+6.062 \times \phi_{2}^{1.252} \times R e^{1.594} \times \phi_{1}^{1.157} \times \operatorname{Pr}^{1.684} \times M^{0.368} \\
\times \lambda^{0.028}\end{array}$ & $\phi_{2}, R e, \phi_{1}, \operatorname{Pr}, M, \lambda$ & 0.4383 \\
\hline
\end{tabular}

Table 9. Correlations for the non-dimensional shear stress.

\begin{tabular}{|c|c|c|}
\hline Correlation for the non-dimensional shear stress & Parameters & $\begin{array}{l}\text { Mean } \\
\text { absolute } \\
\text { error }\end{array}$ \\
\hline$\frac{\sigma . a}{4 \mu_{f} \bar{k} z}=4.294+12.649 \times \phi_{1}{ }^{1.620}$ & $\phi_{1}$ & 1.843 \\
\hline$\frac{\sigma . a}{4 \mu_{f} \bar{k} z}=4.186+349.156 \times \phi_{1}^{1.427} \times \phi_{2}^{1.502}$ & $\phi_{1}, \phi_{2}$ & 1.718 \\
\hline$\frac{\sigma . a}{4 \mu_{f} \bar{k} z}=4.572+158.364 \times \phi_{1}^{3.335} \times \phi_{2}^{3.418} \times R e^{2.961}$ & $\phi_{1}, \phi_{2}, R e$ & 1.174 \\
\hline$\frac{\sigma . a}{4 \mu_{f} \bar{k} z}=4.540+63.787 \times \phi_{1}^{2.823} \times \phi_{2}^{2.894} \times R e^{2.642} \times \lambda^{0.003}$ & $\phi_{1}, \phi_{2}, R e, \lambda$ & 1.145 \\
\hline$\frac{\sigma . a}{4 \mu_{f} \bar{k} Z}=4.511+46.080 \times \phi_{1}^{2.556} \times \phi_{2}^{2.618} \times R e^{2.470} \times \lambda^{0.061} \times \operatorname{Pr}^{2.715}$ & $\phi_{1}, \phi_{2}, R e, \lambda, P r$ & 0.633 \\
\hline $\begin{aligned} \frac{\sigma . a}{4 \mu_{f} \bar{k} z}=4.458 & +421.910 \times \phi_{1}{ }^{2.142} \times \phi_{2}{ }^{2.225} \times R e^{2.228} \times \lambda^{0.189} \times \operatorname{Pr}^{2.429} \\
& \times M^{-2.702}\end{aligned}$ & $\phi_{1}, \phi_{2}, R e, \lambda, \operatorname{Pr}$ & 0.584 \\
\hline
\end{tabular}




\section{Conclusions}

We have numerically investigated the heat convection and thermodynamic irreversibilities of a flow of $\mathrm{Al}_{2} \mathrm{O}_{3}-\mathrm{Cu}$-water hybrid nanofluid moving around a cylinder in porous media. A similarity solution aided by a finite difference scheme was used to solve for the flow and thermal fields. Although this approach gave accurate results, the large number of variables and their mutual interactions complicated the problem. Consequently, a parametric study would have required a large number of simulations to be performed. Instead, we applied a data-driven predictor that leverages machine learning to develop correlations for the flow and heat transfer characteristics. Numerical data were generated and then fed to a multi-layer perception neural network. After the network was trained, the response of the heat transfer and irreversibility to variations in the control parameters was examined. The predictions showed that decreasing the porosity or increasing the Reynolds number led to enhanced heat transfer via intensification of the forced convection process. Similar changes were observed when increasing the volume fraction of nanoparticles. Spherical-shaped nanoparticles were found to produce higher rates of heat transfer than platelet-shaped nanoparticles. As expected, all the trends associated with enhanced heat transfer were found to reduce the thermal and total irreversibilities. Moreover, it was shown that increasing the porosity of the medium or the Reynolds number led to an increase in shear stress. Machine learning techniques were then further exploited to develop thermohydraulic correlations, which provided a deeper fundamental understanding of the flow physics as well as being useful for engineering design analysis. In particular, particle swarm optimization was used, and correlations were developed for the Nusselt number and the nondimensional shear stress as a function of various control parameters. The present study highlights the potential of using neural networks and particle swarm optimization in predicting the thermodynamic and thermohydraulic characteristics of complex thermal and solutal systems.

\section{References}

[1] Vafai, K. ed., 2015. Handbook of porous media. CRC Press.

[2] M. Torabi, C. Dickson, N. Karimi, 2016 "Theoretical investigation of entropy generation and heat transfer by forced convection of copper-water nanofluid in a porous channel- Local thermal nonequilibrium and partial filling effects", Powder Technology, 301, 234-254. DOI: 10.1016/j.powtec.2016.06.017.

[3] Hunt, G., Torabi, M., Govone, L., Karimi, N., \& Mehdizadeh, A. (2018). Two-dimensional heat and mass transfer and thermodynamic analyses of porous microreactors with Soret and thermal radiation effects-an analytical approach. Chemical Engineering and Processing-Process Intensification, 126, 190-205.

[4] Govone, L., Torabi, M., Wang, L., \& Karimi, N. (2019). Effects of nanofluid and radiative heat transfer on the double-diffusive forced convection in microreactors. Journal of Thermal Analysis and Calorimetry, 135(1), 45-59. 
[5] Guthrie, D. G., Torabi, M., \& Karimi, N. (2019). Energetic and entropic analyses of doublediffusive, forced convection heat and mass transfer in microreactors assisted with nanofluid. Journal of Thermal Analysis and Calorimetry, 137(2), 637-658.

[6] Ting, T.W., Hung, Y.M. and Guo, N., 2015. Entropy generation of viscous dissipative nanofluid convection in asymmetrically heated porous microchannels with solid-phase heat generation. Energy Conversion and Management, 105, pp.731-745.

[7] Hunt, G., Karimi, N., \& Torabi, M. (2017). Analytical investigation of heat transfer and classical entropy generation in microreactors-The influences of exothermicity and asymmetry. Applied Thermal Engineering, 119, 403-424.

[8] Govone, L., Torabi, M., Wang, L., \& Karimi, N. (2019). Effects of nanofluid and radiative heat transfer on the double-diffusive forced convection in microreactors. Journal of Thermal Analysis and Calorimetry, 135(1), 45-59.

[9] A. Saeed, N. Karimi, G. Hunt, M. Torabi, 2019 "On the influence of surface heat release and thermal radiation upon transport in catalytic porous microreactors-A novel porous-solid interface model", Chemical Engineering and Processing-Process Intensification, 143, 107602. https://doi.org/10.1016/j.cep.2019.107602.

[10] Kefayati, G.H.R., 2016. Heat transfer and entropy generation of natural convection on nonNewtonian nanofluids in a porous cavity. Powder Technology, 299, pp.127-149.

[11] Hoseinpour, B., Ashorynejad, H.R. and Javaherdeh, K., 2017. Entropy generation of nanofluid in a porous cavity by lattice Boltzmann method. Journal of Thermophysics and Heat Transfer, 31(1), pp.20-27.

[12] Siavashi, M., Yousofvand, R. and Rezanejad, S., 2018. Nanofluid and porous fins effect on natural convection and entropy generation of flow inside a cavity. Advanced Powder Technology, 29(1), pp.142-156.

[13] Baghsaz, S., Rezanejad, S. and Moghimi, M., 2019. Numerical investigation of transient natural convection and entropy generation analysis in a porous cavity filled with nanofluid considering nanoparticles sedimentation. Journal of Molecular Liquids, 279, pp.327-341.

[14] Hussain, S., Mehmood, K., Sagheer, M. and Yamin, M., 2018. Numerical simulation of double diffusive mixed convective nanofluid flow and entropy generation in a square porous enclosure. International Journal of Heat and Mass Transfer, 122, pp.1283-1297.

[15] Hosseini, S.R., Ghasemian, M., Sheikholeslami, M., Shafee, A. and Li, Z., 2019. Entropy analysis of nanofluid convection in a heated porous microchannel under MHD field considering solid heat generation. Powder technology, 344, pp.914-925.

[16] Ibáñez, G., López, A., Pantoja, J. and Moreira, J., 2016. Entropy generation analysis of a nanofluid flow in MHD porous microchannel with hydrodynamic slip and thermal radiation. International Journal of Heat and Mass Transfer, 100, pp.89-97.

[17] Lopez, A., Ibanez, G., Pantoja, J., Moreira, J. and Lastres, O., 2017. Entropy generation analysis of MHD nanofluid flow in a porous vertical microchannel with nonlinear thermal radiation, slip flow and convective-radiative boundary conditions. International Journal of Heat and Mass Transfer, 107, pp.982-994.

[18] Goqo, S.P., Mondal, H., Sibanda, P. and Motsa, S.S., 2019. A multivariate spectral quasilinearisation method for entropy generation in a square cavity filled with porous medium saturated by nanofluid. Case Studies in Thermal Engineering, 14, p.100415. 
[19] Mansour, M.A., Siddiqa, S., Gorla, R.S.R. and Rashad, A.M., 2018. Effects of heat source and sink on entropy generation and MHD natural convection of $\mathrm{Al} 2 \mathrm{O} 3-\mathrm{Cu} /$ water hybrid nanofluid filled with square porous cavity. Thermal Science and Engineering Progress, 6, pp.57-71.

[20] Alizadeh, R., Karimi, N., \& Nourbakhsh, A. (2019). Effects of radiation and magnetic field on mixed convection stagnation-point flow over a cylinder in a porous medium under local thermal nonequilibrium. Journal of Thermal Analysis and Calorimetry, 1-21.

[21] Alizadeh, R., Gomari, S. R., Alizadeh, A., Karimi, N., \& Li, L. K. B. (2019). Combined heat and mass transfer and thermodynamic irreversibilities in the stagnation-point flow of Casson rheological fluid over a cylinder with catalytic reactions and inside a porous medium under local thermal nonequilibrium. Computers \& Mathematics with Applications.

[22] Rashidi, M.M., Ali, M., Freidoonimehr, N. and Nazari, F., 2013. Parametric analysis and optimization of entropy generation in unsteady MHD flow over a stretching rotating disk using artificial neural network and particle swarm optimization algorithm. Energy, 55, pp.497-510.

[23] Siavashi, M., Bahrami, H.R.T. and Aminian, E., 2018. Optimization of heat transfer enhancement and pumping power of a heat exchanger tube using nanofluid with gradient and multilayered porous foams. Applied Thermal Engineering, 138, pp.465-474.

[24] Shahsavar, A., Moradi, M. and Bahiraei, M., 2018. Heat transfer and entropy generation optimization for flow of a non-Newtonian hybrid nanofluid containing coated $\mathrm{CNT} / \mathrm{Fe} 3 \mathrm{O} 4$ nanoparticles in a concentric annulus. Journal of the Taiwan Institute of Chemical Engineers, 84, pp.28-40.

[25] Bahiraei, M., Nazari, S., Moayedi, H. and Safarzadeh, H., 2020. Using neural network optimized by imperialist competition method and genetic algorithm to predict water productivity of a nanofluidbased solar still equipped with thermoelectric modules. Powder Technology, 366, pp.571-586.

[26] Keykhah, S., Assareh, E., Moltames, R., Izadi, M. and Ali, H.M., 2019. Heat transfer and fluid flow for tube included a porous media: Assessment and Multi-Objective Optimization Using Particle Swarm Optimization (PSO) Algorithm. Physica A: Statistical Mechanics and its Applications, p.123804.

[27] Abad, J.M.N., Alizadeh, R., Fattahi, A., Doranehgard, M.H., Alhajri, E. and Karimi, N., 2020. Analysis of transport processes in a reacting flow of hybrid nanofluid around a bluff-body embedded in porous media using artificial neural network and particle swarm optimization. Journal of Molecular Liquids, p.113492.

[28] Alizadeh, R., Mohebbi Najm Abad, J., Fattahi, A., Alhajri, E.S. and Karimi, N., 2020. Application of Machine Learning to Investigation of Heat and Mass Transfer Over a Cylinder Surrounded by Porous Media-The Radial Basic Function Network. Journal of Energy Resources Technology, pp.1-18.

[29] Ebrahimi-Moghadam, A., Mohseni-Gharyehsafa, B. and Farzaneh-Gord, M., 2018. Using artificial neural network and quadratic algorithm for minimizing entropy generation of A12O3-EG/W nanofluid flow inside parabolic trough solar collector. Renewable energy, 129, pp.473-485.

[30] Alizadeh, Rasool, et al. "On the hydrodynamics and heat convection of an impinging external flow upon a cylinder with transpiration and embedded in a porous medium." Transport in Porous Media 120.3 (2017): 579-604.

[31] Alizadeh, R., Rahimi, A.B., Arjmandzadeh, R., Najafi, M. and Alizadeh, A., 2016. Unaxisymmetric stagnation-point flow and heat transfer of a viscous fluid with variable viscosity on a cylinder in constant heat flux. Alexandria Engineering Journal, 55(2), pp.1271-1283. 
[32] Gomari, S. R., Alizadeh, R., Alizadeh, A., \& Karimi, N. (2019). Generation of entropy during forced convection of heat in nanofluid stagnation-point flows over a cylinder embedded in porous media. Numerical Heat Transfer, Part A: Applications, 75(10), 647-673.

[33] Tahmasebi, A., Mahdavi, M. and Ghalambaz, M., 2018. Local thermal nonequilibrium conjugate natural convection heat transfer of nanofluids in a cavity partially filled with porous media using Buongiorno's model. Numerical Heat Transfer, Part A: Applications, 73(4), pp.254-276.

[34] Cunning, G.M., Davis, A.M.J. and Weidman, P.D., 1998. Radial stagnation flow on a rotating circular cylinder with uniform transpiration. Journal of Engineering mathematics, 33(2), pp.113-128.

[35] Thomas, J.W., 2013. Numerical partial differential equations: finite difference methods (Vol. 22). Springer Science \& Business Media.

[36] Ganesan, P. and Palani, G., 2004. Finite difference analysis of unsteady natural convection MHD flow past an inclined plate with variable surface heat and mass flux. International journal of heat and mass transfer, 47(19-20), pp.4449-4457.

[37] Ghadikolaei, S. S., Yassari, M., Sadeghi, H., Hosseinzadeh, K., \& Ganji, D. D., 2017. Investigation on thermophysical properties of Tio2- $\mathrm{Cu} / \mathrm{H} 2 \mathrm{O}$ hybrid nanofluid transport dependent on shape factor in MHD stagnation point flow. Powder technology, 322, pp. 428-438.

[38] Bergman, T.L., Incropera, F.P., Lavine, A.S. and DeWitt, D.P., 2011. Introduction to heat transfer. John Wiley \& Sons.

[39] Alizadeh, R., Rahimi, A.B., Karimi, N. and Alizadeh, A., 2017. On the hydrodynamics and heat convection of an impinging external flow upon a cylinder with transpiration and embedded in a porous medium. Transport in Porous Media, 120(3), pp.579-604.

[40] Torabi, M., Karimi, N., Peterson, G.P. and Yee, S., 2017. Challenges and progress on the modelling of entropy generation in porous media: a review. International Journal of Heat and Mass Transfer, 114, pp.31-46.

[41] Torabi, M., Zhang, K., Karimi, N. and Peterson, G.P., 2016. Entropy generation in thermal systems with solid structures-a concise review. International Journal of Heat and Mass Transfer, 97, pp.917-931.

[42] Saleh, R. and Rahimi, A.B., 2004. Axisymmetric stagnation-point flow and heat transfer of a viscous fluid on a moving cylinder with time-dependent axial velocity and uniform transpiration. $J$. Fluids Eng., 126(6), pp.997-1005.

[43] Drucker, H., Burges, C.J., Kaufman, L., Smola, A.J. and Vapnik, V., 1997. "Support vector regression machines,” Adv Neural Inf Process Syst .,pp. 155-161.

[44] Strutz, T., 2010. Data fitting and uncertainty. A practical introduction to weighted least squares and beyond. Vieweg+ Teubner.

[45] Broomhead, D.S. and Lowe, D., 1988. Radial basis functions, multi-variable functional interpolation and adaptive networks (No. RSRE-MEMO-4148). Royal Signals and Radar Establishment Malvern (United Kingdom).

[46] Hassoun, M.H., 1995. Fundamentals of artificial neural networks. MIT press.

[47] Alanis, A.Y., Arana-Daniel, N. and Lopez-Franco, C. eds., 2019. Artificial Neural Networks for Engineering Applications. Academic Press. 
[48] AlNuaimi, N., Masud, M. M., Serhani, M. A., \& Zaki, N., 2019. Streaming feature selection algorithms for big data: A survey. Applied Computing and Informatics.

[49] Sakar, C.O., Kursun, O. and Gurgen, F., 2012. A feature selection method based on kernel canonical correlation analysis and the minimum Redundancy-Maximum Relevance filter method. Expert Systems with Applications, 39(3), pp.3432-3437.

[50] Parsopoulos, K.E. and Vrahatis, M.N., 2010. Particle swarm optimization and intelligence: advances and applications.

[51] Gorla, R.S.R., 1976. Heat transfer in an axisymmetric stagnation flow on a cylinder. Applied Scientific Research, 32(5), pp.541-553.

[52] Sibalija, T.V., 2019. Particle swarm optimisation in designing parameters of manufacturing processes: A review (2008-2018). Applied Soft Computing, 84, p.105743.

[53] Chan, F. and Tiwari, M. eds., 2007. Swarm Intelligence: focus on ant and particle swarm optimization. BoD-Books on Demand. 